\title{
High Order Relativistic Corrections To Keplerian Motion
}

\author{
L. Fernández-Jambrina \\ Departamento de Enseñanzas Básicas de la Ingeniería Naval \\ E.T.S.I. Navales \\ Arco de la Victoria s/n \\ E-28040-Madrid, Spain \\ and \\ C. Hoenselaers \\ Department of Mathematical Sciences \\ Loughborough University of Technology \\ Loughborough LE11 3TU, United Kingdom
}

PACS numbers: $04.25 \mathrm{Nx}, 95.10 . \mathrm{Ce}$

\begin{abstract}
The first terms of the general solution for an asymptotically flat stationary axisymmetric vacuum spacetime endowed with an equatorial symmetry plane are calculated from the corresponding Ernst potential up to seventh order in the radial pseudospherical coordinate. The metric is used to determine the influence of high order multipoles in the perihelion precession of an equatorial orbit and in the node line precession of a nonequatorial orbit with respect to a geodesic circle. Both results are written in terms of invariant quantities such as the Geroch-Hansen multipoles and the energy and angular momentum of the orbit.
\end{abstract}




\section{Introduction}

The construction of new exact solutions of the Einstein vacuum field equations describing an asymptotically flat stationary axially symmetric spacetime has increased in the last decade due to the several methods of generation of solutions from a given one, such as the Bäcklund transformations, the inverse scattering method and the HKX tranformations (cf. for instance 1 and references quoted therein.) Nevertheless we are very far from being able to implement the physical behaviour of an exact solution at will and in order to obtain results which could be tested experimentally we are led to use approximate expressions for the metrics with the desired physical requirements.

In this paper we study the influence of the first Geroch-Hansen multipole moments [2], 3] of order higher than three in some astrophysical situations with stationary and axial symmetry. As it is well known, these moments can be calculated from the coefficients of the power expansion of the Ernst potential on the symmetry axis [4, being linear the relation between both families of constants until the third moment, that is, the octupole. The subsequent expressions for the multipole moments of order higher than three become considerably more complicated as the order increases and there is not even a closed formula for calculating all of them. The purpose of this paper is therefore to show to what extent these nonlinearities affect the motion of test particles tracing their orbits around a non-spherical, in principle rotating, compact mass distribution. Of course, these terms are irrelevant for our solar system calculations, but they are meaningful for highly relativistic astrophysical objects, such as pulsars, as stated in [5].

With this aim in mind we calculate in section [1] the first seven terms of a power expansion of the Ernst potential with arbitrary values for the multipole moments and construct the corresponding approximate metric. This result is used in section III to obtain an expression for the perihelion precession of an equatorial trajectory and in section IV to produce the corrections to the Newtonian precession of the nodes of a slightly non-equatorial orbit with reference to a close neighbouring geodesic circle. There is some previous work on this subject in [6] and [7, but these references deal only with terms up to the quadrupole moment. The results will be discussed in section $[\nabla$

\section{Calculation of the metric}

The metric of a stationary axially symmetric vacuum spacetime can be written in a canonical form in terms of the Weyl coordinates,

$$
d s^{2}=-f(d t-A d \phi)^{2}+\frac{1}{f}\left\{e^{2 \gamma}\left(d \rho^{2}+d z^{2}\right)+\rho^{2} d \phi^{2}\right\},
$$

where $t$ and $\phi$ are the coordinates associated with the commuting Killing vectors $\partial_{t}$ and $\partial_{\phi}$, and the functions $f, A$ and $\gamma$ depend only on the coordinates $\rho$ and $z$. 
The whole set of Einstein equations can be shown $[8$ to be equivalent to the following system of partial differential equations,

$$
\begin{gathered}
\varepsilon=f+i \chi \\
\varepsilon_{\rho \rho}+\frac{1}{\rho} \varepsilon_{\rho}+\varepsilon_{z z}=\frac{2}{\varepsilon+\bar{\varepsilon}}\left(\varepsilon_{\rho}^{2}+\varepsilon_{z}{ }^{2}\right) \\
A_{\rho}=\frac{4 \rho}{(\varepsilon+\bar{\varepsilon})^{2}} \chi_{z} \\
A_{z}=-\frac{4 \rho}{(\varepsilon+\bar{\varepsilon})^{2}} \chi_{\rho} \\
\gamma_{\rho}=\frac{\rho}{(\varepsilon+\bar{\varepsilon})^{2}}\left(\varepsilon_{\rho} \bar{\varepsilon}_{\rho}-\varepsilon_{z} \bar{\varepsilon}_{z}\right) \\
\gamma_{z}=\frac{\rho}{(\varepsilon+\bar{\varepsilon})^{2}}\left(\varepsilon_{\rho} \bar{\varepsilon}_{z}+\varepsilon_{z} \bar{\varepsilon}_{\rho}\right) .
\end{gathered}
$$

It can be shown that the integrability of the last four equations is guaranteed if equation (3), the Ernst equation, is satisfied. Therefore, in order to obtain a solution of the Einstein equations with two Killing vectors, it suffices to solve the Ernst equation and then calculate the metric functions by quadratures.

It is also usual to write the Ernst equation in terms of another potential, $\xi$, related to the previous one by the following relation,

$$
\xi=\frac{1-\varepsilon}{1+\varepsilon},
$$

which satisfies another partial differential equation,

$$
(\xi \bar{\xi}-1)\left(\xi_{\rho \rho}+\frac{1}{\rho} \xi_{\rho}+\xi_{z z}\right)=2 \bar{\xi}\left(\xi_{\rho}^{2}+\xi_{z}{ }^{2}\right),
$$

which is also satisfied by $\xi^{-1}$. This latter form is the one introduced originally in [8] and allows a simple integration of the Kerr metric.

This form is particularly useful for calculating the multipole moments and it will be the one we shall employ.

In order to calculate the approximate solution, we shall write the Ernst potential $\xi$ as a function of two coordinates $r$ and $\theta$ related to the Weyl ones by the following transformation,

$$
\rho=r \sin \theta \quad z=r \cos \theta .
$$

We can implement the requirement of asymptotic flatness by writing $\xi$ as a formal inverse power expansion in the pseudospherical radial coordinate $r$,

$$
\xi=\sum_{n=1}^{\infty} \xi_{n} r^{-n}
$$


where the functions $\xi_{n}$ depend only on the coordinate $\theta$.

Since we are interested in having a solution which is symmetric with respect to the equatorial plane $\theta=\pi / 2$, we shall require that the functions $\xi_{n}$ of odd order be real whereas those of even order will be taken to be imaginary.

With this information at hand we can now proceed to calculate the metric functions. The function $f$ is just the real part of $\varepsilon$ and from our knowledge of $\xi$ we can calculate the first eight terms of its expansion in the radial coordinate,

$$
\begin{aligned}
& f=1+\sum_{n=1}^{\infty} f_{n} r^{-n} \\
& f_{1}=-2 m_{0} \\
& f_{2}=2 m_{0}^{2} \\
& f_{3}=m_{2}-\frac{4 m_{0}^{3}}{3}-3 m_{2} \cos ^{2} \theta \\
& f_{4}=-2 m_{0} m_{2}+\frac{2 m_{0}^{4}}{3}+\left(6 m_{0} m_{2}+2 m_{1}^{2}\right) \cos ^{2} \theta \\
& f_{5}=-\frac{3 m_{4}}{4}+2 m_{0}^{2} m_{2}+\frac{8 m_{0} m_{1}^{2}}{35}-\frac{4 m_{0}^{5}}{15}+ \\
& +\left(\frac{15 m_{4}}{2}-6 m_{0}^{2} m_{2}-\frac{44 m_{0} m_{1}^{2}}{7}\right) \cos ^{2} \theta-\frac{35 m_{4} \cos ^{4} \theta}{4} \\
& f_{6}=\frac{3 m_{0} m_{4}}{2}+\frac{m_{2}^{2}}{2}-\frac{4 m_{2} m_{0}^{3}}{3}-\frac{16 m_{0}^{2} m_{1}^{2}}{35}+\frac{4 m_{0}^{6}}{45}+ \\
& +\left(-15 m_{0} m_{4}-6 m_{1} m_{3}-3 m_{2}^{2}+4 m_{2} m_{0}^{3}+\frac{356 m_{0}^{2} m_{1}^{2}}{35}\right) \cos ^{2} \theta+ \\
& +\left(\frac{35 m_{0} m_{4}}{2}+10 m_{1} m_{3}+\frac{9 m_{2}^{2}}{2}\right) \cos ^{4} \theta \\
& f_{7}=\frac{5 m_{6}}{8}-\frac{3 m_{0}^{2} m_{4}}{2}-\frac{4 m_{0} m_{1} m_{3}}{11}-m_{0} m_{2}^{2}-\frac{16 m_{1}^{2} m_{2}}{231}+ \\
& +\frac{2 m_{0}^{4} m_{2}}{3}+\frac{32 m_{0}^{3} m_{1}^{2}}{63}-\frac{8 m_{0}{ }^{7}}{315}+
\end{aligned}
$$




$$
\begin{aligned}
& +\left(-\frac{105 m_{6}}{8}+15 m_{0}^{2} m_{4}+\frac{216 m_{0} m_{1} m_{3}}{11}+6 m_{0} m_{2}^{2}+\right. \\
& \left.+\frac{38 m_{1}^{2} m_{2}}{11}-2 m_{0}^{4} m_{2}-\frac{1208 m_{0}^{3} m_{1}^{2}}{105}\right) \cos ^{2} \theta+ \\
& +\left(\frac{315 m_{6}}{8}-\frac{35 m_{0}^{2} m_{4}}{2}-\frac{340 m_{0} m_{1} m_{3}}{11}-\frac{114 m_{1}^{2} m_{2}}{11}-\right. \\
& \left.-9 m_{0} m_{2}^{2}\right) \cos ^{4} \theta-\frac{231 m_{6}}{8} \cos ^{6} \theta
\end{aligned}
$$

where the constants $m_{n}$ which arise from the integration of the Ernst equation are real if $n$ is even and otherwise imaginary.

The first six terms of the metric function $A$ can be obtained by direct integration of the equations (4) and (5). In spite of the factor $i$ before the expression for $A$, this function is obviously real since the constants $m_{n}$ are imaginary for odd $n$,

$$
\begin{gathered}
A=-i \sin ^{2} \theta \sum_{n=1}^{\infty} A_{n} r^{-n} \\
A_{1}=-2 m_{1} \\
A_{2}=-2 m_{0} m_{1} \\
A_{3}=m_{3}-\frac{8 m_{0}{ }^{2} m_{1}}{5}-5 m_{3} \cos ^{2} \theta \\
=\frac{3 m_{0} m_{3}}{2}+\frac{m_{1} m_{2}}{2}-\frac{16 m_{0}{ }^{3} m_{1}}{15}+ \\
+\left(-\frac{15 m_{0} m_{3}}{2}+\frac{3 m_{1} m_{2}}{2}\right) \cos ^{2} \theta \\
=-\frac{3 m_{5}}{4}+\frac{4 m_{0}{ }^{2} m_{3}}{3}+\frac{8 m_{0} m_{1} m_{2}}{7}-\frac{64 m_{0}{ }^{4} m_{1}}{105}+ \\
+\left(\frac{21 m_{5}}{2}-\frac{20 m_{0}{ }^{2} m_{3}}{3}\right) \cos ^{2} \theta-\frac{63 m_{5}}{4} \cos ^{4} \theta \\
A_{5}=\frac{5 m_{0} m_{5}}{4}-\frac{m_{1} m_{4}}{4}-\frac{m_{2} m_{3}}{2}+\frac{8 m_{0}{ }^{3} m_{3}}{9}+ \\
+\frac{48 m_{0}{ }^{2} m_{1} m_{2}}{35}+\frac{8 m_{0} m_{1}{ }^{3}}{105}-\frac{32 m_{0}{ }^{5} m_{1}}{105}+\left(\frac{35 m_{0} m_{5}}{2}-\frac{5 m_{1} m_{4}}{2}+\right.
\end{gathered}
$$




$$
\begin{aligned}
& \left.+m_{2} m_{3}-\frac{40 m_{0}{ }^{3} m_{3}}{9}-\frac{8 m_{0}^{2} m_{1} m_{2}}{5}+\frac{16 m_{0} m_{1}{ }^{3}}{21}\right) \cos ^{2} \theta+ \\
& +\quad\left(-\frac{105 m_{0} m_{5}}{4}+\frac{35 m_{1} m_{4}}{4}-\frac{5 m_{2} m_{3}}{2}\right) \cos ^{4} \theta
\end{aligned}
$$

The only function which remains to be calculated is $\gamma$ and can be obtained as a quadrature from equations (6) and (7),

$$
\begin{aligned}
& \gamma=\sum_{n=1}^{\infty} \gamma_{n} r^{-2 n} \\
& \gamma_{1}=-\frac{m_{0}^{2} \sin ^{2} \theta}{2} \\
& \gamma_{2}=\frac{3 m_{0} m_{2}}{4}-\frac{m_{1}^{2}}{4}+\left(-\frac{9 m_{0} m_{2}}{2}+\frac{5 m_{1}^{2}}{2}\right) \cos ^{2} \theta+ \\
& +\left(\frac{15 m_{0} m_{2}}{4}-\frac{9 m_{1}^{2}}{4}\right) \cos ^{4} \theta \\
& \gamma_{3}=-\frac{5 m_{0} m_{4}}{8}+\frac{m_{1} m_{3}}{2}-\frac{3 m_{2}^{2}}{8}+\frac{2 m_{0}^{2} m_{1}^{2}}{35}+ \\
& +\left(\frac{75 m_{0} m_{4}}{8}-\frac{21 m_{1} m_{3}}{2}+\frac{45 m_{2}^{2}}{8}-\frac{2 m_{0}^{2} m_{1}^{2}}{35}\right) \cos ^{2} \theta+ \\
& +\left(-\frac{175 m_{0} m_{4}}{8}+\frac{55 m_{1} m_{3}}{2}-\frac{117 m_{2}^{2}}{8}\right) \cos ^{4} \theta+ \\
& +\left(\frac{105 m_{0} m_{4}}{8}-\frac{35 m_{1} m_{3}}{2}+\frac{75 m_{2}^{2}}{8}\right) \cos ^{6} \theta \\
& \gamma_{4}=\frac{35 m_{0} m_{6}}{64}-\frac{15 m_{1} m_{5}}{32}+\frac{45 m_{2} m_{4}}{64}- \\
& -\frac{9 m_{3}^{2}}{32}-\frac{14 m_{0}^{2} m_{1} m_{3}}{165}-\frac{2 m_{0} m_{1}^{2} m_{2}}{33}+\frac{16 m_{0}^{4} m_{1}^{2}}{1575}+ \\
& +\left(-\frac{245 m_{0} m_{6}}{16}+\frac{135 m_{1} m_{5}}{8}-\frac{315 m_{2} m_{4}}{16}+\frac{81 m_{3}^{2}}{8}+\right. \\
& \left.+\quad \frac{28 m_{0}^{2} m_{1} m_{3}}{55}-\frac{4 m_{0} m_{1}^{2} m_{2}}{231}+\frac{16 m_{0}^{4} m_{1}^{2}}{1575}\right) \cos ^{2} \theta+ \\
& +\left(\frac{2205 m_{0} m_{6}}{32}-\frac{1365 m_{1} m_{5}}{16}+\frac{3075 m_{2} m_{4}}{32}-\right. \\
& \left.-\quad \frac{795 m_{3}^{2}}{16}-\frac{14 m_{0}^{2} m_{1} m_{3}}{33}+\frac{6 m_{0} m_{1}^{2} m_{2}}{77}\right) \cos ^{4} \theta+
\end{aligned}
$$




$$
\begin{aligned}
& +\left(-\frac{1617 m_{0} m_{6}}{16}+\frac{1071 m_{1} m_{5}}{8}-\frac{2415 m_{2} m_{4}}{16}+\frac{625 m_{3}^{2}}{8}\right) \cos ^{6} \theta+ \\
& +\left(\frac{3003 m_{0} m_{6}}{64}-\frac{2079 m_{1} m_{5}}{32}+\frac{4725 m_{2} m_{4}}{64}-\frac{1225 m_{3}^{2}}{32}\right) \cos ^{8} \theta .
\end{aligned}
$$

\section{Perihelion precession of a closed orbit}

It is well known from Bertrand's theorem (cf. for instance 9]) that stable bounded orbits of particles moving under the influence of a central force which is neither Newtonian nor harmonic are not closed. Therefore whenever the source of the gravitational field is not exactly monopolar, the bounded trajectories on the equatorial plane will no longer be the elliptic orbits described by Kepler's first law but will take the form of a precessing ellipse if the deviation from spherical symmetry is small.

The situation becomes a bit more complicated in general relativity. Although it has been proven [10] that there are just two asymptotically flat static spherically symmetric spacetimes in which the stable orbits are closed, they are rather different from the classical physical situations. Therefore, when relativistic effects are taken into account, not even the motion around a spherical distribution of mass is closed. This effect has been tested in our solar system and it amounts to a slow precession of the perihelion of the orbit of Mercury. Of course other multipole moments of the mass distribution will also contribute to this effect and, in principle, these moments could be calculated by measuring the precession of a certain number of test particles orbiting at conveniently different distances from the gravitational source.

If the test particles are small enough for the tidal forces to be unimportant within the characteristic length of the particle, we can regard them as point particles. If the effects due to their intrinsic angular momentum can be taken as negligible it can be assumed that they trace out timelike geodesics in the spacetime surrounding the gravitational source. Hence, in order to study the influence of the far field multipole moments of the gravitational field, we shall have to solve the geodesic equations for the previously calculated metric. We shall restrict ourselves to the equatorial plane $\theta=\pi / 2$.

Since the timelike and azimuthal coordinates are ignorable, we have two first integrals for the motion corresponding to the conserved quantities $E$ and $l$, respectively the total energy per unit of mass and the projection of the angular momentum on the $z$ axis, also per unit of mass, of the test particle. In terms of its 4 -velocity $u=(\dot{t}, \dot{r}, \dot{\theta}, \dot{\phi})$ these quantities have the following form,

$$
\begin{gathered}
E=-\partial_{t} \cdot u=f(\dot{t}-A \dot{\phi}) \\
l=\partial_{\phi} \cdot u=f A(\dot{t}-A \dot{\phi})+\frac{1}{f} r^{2} \dot{\phi},
\end{gathered}
$$

where the overhead dot stands for the derivative with respect to proper time.

Therefore the equations for $t$ and $\phi$ can be written as follows, 


$$
\begin{gathered}
\dot{\phi}=f \frac{l-E A}{r^{2}} \\
\dot{t}=\frac{E}{f}+f A \frac{l-E A}{r^{2}} .
\end{gathered}
$$

Another integral arises from the fact that the trajectory is timelike and therefore $u \cdot u=-1$. For the geodesics under consideration this means,

$$
-1=-f(\dot{t}-A \dot{\phi})^{2}+\frac{1}{f}\left(e^{2 \gamma} \dot{r}^{2}+r^{2} \dot{\phi}^{2}\right) .
$$

From the previous three equations $\dot{r}$ can be obtained as a function of the non-ignorable coordinates and the conserved quantities. However, since we are interested in the shape of the orbit rather than in its time evolution, we divide (36) by $\dot{\phi}$ to get the derivative of the radial coordinate with respect to the azimuthal angle,

$$
r_{\phi}^{2}=e^{-2 \gamma}\left\{\frac{r^{4}\left(E^{2}-f\right)}{f^{2}(l-E A)^{2}}-r^{2}\right\} .
$$

It will be useful to write this equation in terms of another function $u=1 / r$ as it is done in classical mechanics for solving the motion under central forces,

$$
u_{\phi}^{2}=e^{-2 \gamma}\left\{\frac{E^{2}-f}{f^{2}(l-E A)^{2}}-u^{2}\right\}=F(u)=\sum_{n=0}^{6} c_{n} u^{n}+O\left(u^{7}\right) .
$$

This equation can be turned into a quasilinear one by taking a derivative with respect to $\phi$ and cancelling the $u_{\phi}$ factors, since for the analysis of perihelion precession circular orbits are of no interest,

$$
u_{\phi \phi}=\frac{1}{2} F^{\prime}(u) .
$$

In order to solve these equations perturbatively we need expand them in powers of a small parameter. A good candidate is the inverse of the angular momentum per unit of mass, $l$, since according to Kepler's 1-2-3 law, which is assumed to be a good approximation at a great distance from the source, it behaves as $l \sim \sqrt{m r}$, where $m$ is the mass of the particle. It can be combined with the mass of the source $m_{0}$ to yield an acceptable dimensionless small parameter for analysing the far gravitational field. Hence we shall use $\epsilon=m_{0} / l$ and expand $u$ in the following way,

$$
u=\epsilon^{2} \sum_{n=0}^{11} u_{n} \epsilon^{n}+O\left(\epsilon^{14}\right) .
$$

The reason for starting the expansion at this order is that the expression for the Kepler ellipse, which is expected to be the first term, is second order in $\epsilon$. 
The energy per unit of mass of the particle is also to be expanded in $\epsilon$. Therefore we write,

$$
E=1+\epsilon^{2} \sum_{n=0}^{n=11} E_{n} \epsilon^{n}+O\left(\epsilon^{14}\right) .
$$

In order to avoid the appearance of secular terms we use a coordinate $\psi$ related to $\phi$ by,

$$
\psi=\omega \phi \quad \omega=\sqrt{1+\sum \omega_{i} \epsilon^{i}} .
$$

The coefficients $c_{n}$ are all of the order $\epsilon^{2}$ except $c_{2}$ which is clearly of zeroth order in $\epsilon$ and therefore equation (38) takes the form of a hierarchy of forced harmonic oscillators which can be solved iteratively up to the order of accuracy provided by our knowledge of the metric,

$$
u_{n \psi \psi}+u_{n}=f_{n}(\psi) .
$$

The first terms of the expansion of the solution to the equations (38) and (39) are,

$$
\begin{gathered}
u_{0}=\frac{1}{m_{0}}\left(1+\sqrt{1+2 E_{0}} \cos \psi\right) \\
u_{1}=0 \\
u_{2}=\frac{6+4 E_{0}}{m_{0}} \\
u_{3}=\left(8+4 E_{0}\right) \frac{i m_{1}}{m_{0}^{3}} .
\end{gathered}
$$

Of course the term of lowest order is the Kepler ellipse if $E_{0}$ is negative.

From the information we have about the metric we can calculate $\omega$ up to the eleventh power of $\epsilon$. These terms are just what we need to calculate the expression for the perihelion precession, so we shall focus on them. The expressions of the terms $u_{n}$ are not needed and therefore we shall no enclose them here.

Instead of writing the results as a function of the integration constants $m_{i}$, it will be more useful to write them in terms of the Geroch-Hansen multipole moments, $P_{i}$, the physical interpretation of which is more appealing. Bear in mind that the odd multipole moments are imaginary and have to be multiplied by $-i$ to obtain the usual real expressions $J_{n}$. To calculate these moments we shall make use of the procedure described in [4.

If we have and expansion of the Ernst potential $\xi$ on the symmetry axis in terms of the Weyl coordinate $z$, viz.

$$
\xi(\rho=0)=\sum_{n=0}^{\infty} C_{n} z^{-(n+1)}
$$


then the multipole moments can be calculated as follows,

$$
\begin{gathered}
P_{n}=C_{n} \quad n \leq 3 \\
P_{4}=C_{4}+\frac{1}{7} \bar{C}_{0}\left(C_{1}^{2}-C_{2} C_{0}\right) \\
P_{5}=C_{5}+\frac{1}{3} \bar{C}_{0}\left(C_{2} C_{1}-C_{3} C_{0}\right)+\frac{1}{21} \bar{C}_{1}\left(C_{1}^{2}-C_{2} C_{0}\right) .
\end{gathered}
$$

As a function of these multipole moments the first coefficients in the expansion of the energy per unit of mass, $E$, read,

$$
\begin{gathered}
E_{1}=0 \\
E_{2}=-6-10 E_{0}-\frac{E_{0}^{2}}{2} \\
E_{3}=-\left(8+12 E_{0}\right) \frac{i P_{1}}{P_{0}^{2}} \\
E_{4}=-\frac{47}{4}-20 E_{0}-13 E_{0}^{2}+\frac{E_{0}^{3}}{2}+\left(2+3 E_{0}\right) \frac{P_{2}}{P_{0}{ }^{3}} \\
E_{5}=-\left(56+104 E_{0}+56 E_{0}^{2}\right) \frac{i P_{1}}{P_{0}{ }^{2}},
\end{gathered}
$$

where $E_{0}$, the Keplerian energy, is a free parameter which has $-1 / 2$ as a lower bound, corresponding to a circular orbit.

The frequency $\omega$ is different from one and therefore the orbit is not closed. Between two consecutives perihelion approaches the test particle traces an angle $2 \pi / \omega$. Hence the perihelion has shifted an angle, $\Delta \phi$ given up to the eleventh power of $\epsilon$ by the following expression,

$$
\begin{aligned}
\Delta \phi & =2 \pi\left(\omega^{-1}-1\right)=\pi\left\{\Delta_{0}+\Delta_{1}+\Delta_{2}+\Delta_{4}+\Delta_{8}+\right. \\
& \left.+\Delta_{16}+\Delta_{32}+\Delta_{2 \times 4}+\Delta_{2 \times 8}+\Delta_{2 \times 16}+\Delta_{4 \times 8}\right\}
\end{aligned}
$$

where the shift has been split into different terms according to their origin: The first one, $\Delta_{0}$, comprises the Newtonian contribution to the precession, that is, the terms which remain after taking the classical limit $c \rightarrow \infty$. Of course only the gravitational moments are present since rotation has no influence whatsoever in Newtonian dynamics. Since the speed of light, $c$, and the gravitational 
coupling constant, $G$, have been taken to be one, the terms look rather alike in magnitude. However, if the respective factors are written (a factor $G / c$ for each $\epsilon$, a factor $G / c^{2}$ for each $P_{2 n}$, a factor $G / c^{3}$ for each $P_{2 n+1}$, a factor $c^{-1}$ for each $l$ and a factor $G^{-2}$ for each $E_{0}$ ), the actual magnitude of every term is recovered. For instance, the first Newtonian term has a factor $G^{2}$ and the second a $G^{4}$. For oblate gravitational sources the quadrupole moment, $P_{2}$ is negative, whence it contributes to a positive shift of the perihelion in the first order. In the next order the shift contribution of the quadrupole is, however, always positive. (N.b. $E_{0}$, though negative for bounded orbits, has a lower limit about $-1 / 2$ which does not allow it to overcome the energy-independent term.) On the other hand the sedecimpole $P_{4}$ term bears the opposite sign to the one of the quadrupole,

$$
\Delta_{0}=-\frac{3 P_{2}}{P_{0}{ }^{3}} \epsilon^{4}+\left\{\left(\frac{105}{8}+\frac{45 E_{0}}{4}\right) \frac{P_{4}}{P_{0}{ }^{5}}+\left(\frac{105}{8}+\frac{15 E_{0}}{4}\right) \frac{P_{2}{ }^{2}}{P_{0}{ }^{6}}\right\} \epsilon^{8} .
$$

The second term, $\Delta_{1}$, comprises the contribution to the perihelion precession due to a spherically symmetric mass distribution, i.e. the Schwarzschild effect. It can be calculated exactly in terms of elliptic functions and is of the order of $G^{2} / c^{2}$. The contribution of every order is always positive,

$$
\begin{aligned}
\Delta_{1} & =6 \epsilon^{2}+\left(\frac{105}{2}+15 E_{0}\right) \epsilon^{4}+\left(\frac{975}{2}+165 E_{0}\right) \epsilon^{6}+ \\
& +\left(\frac{159105}{32}+\frac{16725 E_{0}}{8}+\frac{705 E_{0}^{2}}{8}\right) \epsilon^{8}+ \\
& +\left(\frac{1701507}{32}+\frac{216375 E_{0}}{8}+\frac{20115 E_{0}^{2}}{8}\right) \epsilon^{10} .
\end{aligned}
$$

In the term $\Delta_{2}$ we have included the influence of a dipole of rotation on the perihelion shift. It is of the order of $G^{2} / c^{2}$. Since its lower terms in $\epsilon$ are odd, it is sensitive to whether the probe rotates in the same direction as the source does or not. It is positive if the angular momentum of the source and the orbital angular momentum of the probe are antiparallel and negative otherwise. This has a qualitative explanation in the fact that the perihelion shift due to a mass monopole decreases with the angular momentum of the test particle; if the source happens to be rotating and its angular momentum is $J$, then $l$ is replaced in the first order by $l+2 J / r$ as it can be seen in equation (38) after substitution of $P_{1}$ by $i J$, whence the 'effective' $l$ increases if both momenta are parallel and it would be expected that the perihelion advance diminishes. In contrast, the quadratic terms in $P_{1}$ are independent of the direction of rotation and are always positive whence they induce a perihelion advance. The cubic terms in $P_{1}$ behave as the linear ones,

$$
\Delta_{2}=\frac{8 i P_{1} \epsilon^{3}}{P_{0}{ }^{2}}+\left(168+48 E_{0}\right) \frac{i P_{1}}{P_{0}^{2}} \epsilon^{5}-\left(120+24 E_{0}\right) \frac{P_{1}^{2}}{P_{0}{ }^{4}} \epsilon^{6}+
$$




$$
\begin{aligned}
& +\left(2562+1020 E_{0}+36 E_{0}^{2}\right) \frac{i P_{1}}{P_{0}{ }^{2}} \epsilon^{7}- \\
& -\left(\frac{65607}{16}+\frac{44607 E_{0}}{28}+\frac{195 E_{0}^{2}}{4}\right) \frac{P_{1}^{2}}{P_{0}{ }^{4}} \epsilon^{8}+ \\
& +\left\{\left(36046+17640 E_{0}+1356 E_{0}^{2}-16 E_{0}^{3}\right) \frac{i P_{1}}{P_{0}{ }^{2}}-\left(2048+672 E_{0}\right) \frac{i P_{1}^{3}}{P_{0}{ }^{6}}\right\} \epsilon^{9}- \\
& -\left(\frac{10256685}{112}+\frac{1320387 E_{0}}{28}+\frac{118305 E_{0}^{2}}{28}\right) \frac{P_{1}{ }^{2}}{P_{0}{ }^{4}} \epsilon^{10}+ \\
& +\left\{\left(\frac{3927489}{8}+\frac{569361 E_{0}}{2}+\frac{70659 E_{0}^{2}}{2}+174 E_{0}^{3}+15 E_{0}^{4}\right) \frac{i P_{1}}{P_{0}{ }^{2}}-\right. \\
& \left.-\left(\frac{2735961}{28}+\frac{341339 E_{0}}{7}+\frac{27429 E_{0}^{2}}{7}\right) \frac{i P_{1}^{3}}{P_{0}{ }^{6}}\right\} \epsilon^{11} .
\end{aligned}
$$

Under the name $\Delta_{4}$ the relativistic terms depending only on the quadrupole moment, $P_{2}$, and the mass are comprised. The first correction has a factor $G^{4} / c^{2}$ in front of it. As it was to be expected, it does not depend on the direction of rotation and, as its Newtonian counterpart, it is positive for oblate gravitational sources. The quadratic terms as a whole are always positive,

$$
\begin{aligned}
\Delta_{4} & =-\left(90+42 E_{0}\right) \frac{P_{2}}{P_{0}{ }^{3}} \epsilon^{6}-\left(\frac{25383}{16}+\frac{28305 E_{0}}{28}+\frac{375 E_{0}^{2}}{4}\right) \frac{P_{2}}{P_{0}{ }^{3}} \epsilon^{8}+ \\
& +\left\{-\left(\frac{2686203}{112}+\frac{503379 E_{0}}{28}+\frac{80187 E_{0}^{2}}{28}\right) \frac{P_{2}}{P_{0}{ }^{3}}+\right. \\
& \left.+\left(\frac{12471}{16}+519 E_{0}+\frac{165 E_{0}^{2}}{4}\right) \frac{P_{2}{ }^{2}}{P_{0}{ }^{6}}\right\} \epsilon^{10}
\end{aligned}
$$

The symbol $\Delta_{8}$ stands for the corrections to the perihelion shift due to a rotation octupole moment $P_{3}$. The first correction is of the order of $G^{4} / c^{2}$. They bear the same relation to the dipole terms as the quadrupole to the monopole terms: The overall sign changes,

$$
\begin{aligned}
\Delta_{8} & =-\left\{\left(30+24 E_{0}\right) \epsilon^{7}+\left(801+825 E_{0}+138 E_{0}^{2}\right) \epsilon^{9}+\right. \\
& \left.+\left(\frac{28671}{2}+16475 E_{0}+4278 E_{0}^{2}+102 E_{0}^{3}\right) \epsilon^{11}\right\} \frac{i P_{3}}{P_{0}{ }^{4}}
\end{aligned}
$$

The influence of the sedecimpole gravitational moment, $P_{4}$, is included in $\Delta_{16}$ and does not show up until the tenth order of the small parameter. In non-geometrized units it is proportional to $G^{6} / c^{2}$,

$$
\Delta_{16}=\left(\frac{7425}{16}+570 E_{0}+\frac{495 E_{0}^{2}}{4}\right) \frac{P_{4}}{P_{0}{ }^{5}} \epsilon^{10} .
$$

The last multipole moment to be considered is the rotational trigintaduopole moment, $P_{5}$, and it is comprised in $\Delta_{32}$. It is of the eleventh order in $\epsilon$, 


$$
\Delta_{32}=\left(\frac{945}{8}+210 E_{0}+\frac{135 E_{0}^{2}}{2}\right) \frac{i P_{5}}{P_{0}{ }^{6}} \epsilon^{11} .
$$

Now we review the couplings among the different multipole moments other than mass. Up to the order considered there is no coupling between the gravitational moments higher than the mass (except for self-couplings), but there are rotation-rotation couplings and gravitation-rotation couplings. The first one to appear is the dipole-quadrupole coupling, $\Delta_{2 \times 4}$. It has a factor of $G^{4} / c^{2}$ in the lowest order. If both angular momenta are antiparallel and the source is oblate $\left(P_{2}<0\right)$, then the contribution of the bilinear terms is positive. The quadratic terms in the quadrupole $P_{2}$ are again positive if $J$ and $l$ are antiparallel. Finally the quadratic terms in the dipole are positive if the body is oblate,

$$
\begin{aligned}
\Delta_{2 \times 4} & =-\left(90+24 E_{0}\right) \frac{i P_{1} P_{2}}{P_{0}{ }^{5}} \epsilon^{7}-\left(3939+2127 E_{0}+126 E_{0}^{2}\right) \frac{i P_{1} P_{2}}{P_{0}{ }^{5}} \epsilon^{9}+ \\
& +\left(2280+900 E_{0}\right) \frac{P_{1}^{2} P_{2}}{P_{0}{ }^{7}} \epsilon^{10}+\left\{\left(\frac{1419}{2}+303 E_{0}\right) \frac{i P_{1} P_{2}{ }^{2}}{P_{0}{ }^{8}}-\right. \\
& \left.-\left(\frac{2825301}{28}+\frac{507992 E_{0}}{7}+\frac{75765 E_{0}^{2}}{7}+90 E_{0}^{3}\right) \frac{i P_{1} P_{2}}{P_{0}{ }^{5}}\right\} \epsilon^{11}
\end{aligned}
$$

The only rotation-rotation coupling up to this order is between the dipole and octupole moments. It comes under the name of $\Delta_{2 \times 8}$ and it is at least of the order $G^{6} / c^{4}$. Since it is linear on both rotational moments this term is not sensitive to the direction of rotation. It is of the tenth order in $\epsilon$,

$$
\Delta_{2 \times 8}=\left(1068+972 E_{0}+120 E_{0}^{2}\right) \frac{P_{1} P_{3}}{P_{0}{ }^{6}} \epsilon^{10} .
$$

The higher rotation-gravitation couplings involve the rotational dipole and the gravitational sedecimpole, $\Delta_{2 \times 16}$, and the rotational octupole and the gravitational quadrupole, $\Delta_{4 \times 8}$. Both appear first in the eleventh order of perturbation and are at least of the order of $\epsilon^{11}$. The term $\Delta_{2 \times 16}$ is positive if $P_{4}$ is positive and the angular momenta are antiparallel,

$$
\begin{aligned}
\Delta_{2 \times 16} & =\left(\frac{4005}{8}+495 E_{0}+\frac{135 E_{0}^{2}}{2}\right) \frac{i P_{1} P_{4}}{P_{0}{ }^{7}} \epsilon^{11} \\
\Delta_{4 \times 8} & =\left(\frac{1383}{4}+348 E_{0}+45 E_{0}^{2}\right) \frac{i P_{2} P_{3}}{P_{0}{ }^{7}} \epsilon^{11} .
\end{aligned}
$$

It would be of great interest to know the range of applicability of this perturbative expansion. In an appendix at the end of this paper a simpler case is studied: It is shown there for which values of the parameters the expansions are acceptable for the Schwarzschild metric. 


\section{Precession of the line of nodes of a nonequa- torial orbit}

Let us consider now a bounded orbit slightly departing from the equatorial plane. If the mass distribution of the source were spherically symmetric, the equatorial plane would not be at all privileged and the orbit would always intersect it at the same nodes. However this is no longer the situation when the source is not exactly spherical, since then the nodes precess due to the perturbation generated by higher order multipoles. In classical nonrelativistic mechanics the first contribution to the precession of the nodes arises from the quadrupole moment, as it is shown in an appendix at the end of this paper, whereas in general relativity it is the rotational dipole moment the first one to contribute.

In the approximation used in this section we consider a geodesic on the equatorial plane and calculate the evolution of small deviations from it. Since the geodesic equation reads,

$$
\ddot{x}^{\mu}+\Gamma_{\rho \sigma}^{\mu} \dot{x}^{\rho} \dot{x}^{\sigma}=0
$$

it is straightforward that nearby geodesics which deviate from the original geodesic by a vector $\delta^{\mu}$ fulfill,

$$
\ddot{\delta}^{\mu}+2 \Gamma_{\rho \sigma}^{\mu} \dot{\delta}^{\rho} \dot{x}^{\sigma}+\Gamma_{\rho \sigma, \nu}^{\mu} \dot{x}^{\rho} \dot{x}^{\sigma} \delta^{\nu}=0 .
$$

Since we are interested in small deviations from the equatorial plane, we shall focus our attention on the $\theta$ coordinate. Taking into account that the reference geodesic lies on the symmetry plane $\theta=\pi / 2$ and that the first derivatives of the metric with respect to $\theta$ vanish on it, the geodesic deviation equation for $\delta^{\theta}$ reduces to,

$$
\ddot{\delta}^{\theta}-\frac{1}{2} g^{\theta \theta} g_{\rho \sigma, \theta \theta} \dot{x}^{\rho} \dot{x}^{\sigma} \delta^{\theta}=0 .
$$

Instead of considering an arbitrary bounded reference geodesic on the equatorial plane, we shall restrict ourselves to geodesic circles. Of course many interesting features will be lost, but we deem this paper would become even longer if we take them into account.

In order to compute the evolution of the nodes with respect to the azimuthal angle on the geodesic, the previous equation needs to be divided by $\dot{\phi}^{2}$, which is constant on the circle. From now on we shall write $\delta$ instead of $\delta^{\theta}$ to avoid cumbersome notations,

$$
\delta_{\phi \phi}+\Omega^{2} \delta=0 \quad \Omega^{2}=-\frac{1}{2 \dot{\phi}^{2}} g^{\theta \theta} g_{\rho \sigma, \theta \theta} \dot{x}^{\rho} \dot{x}^{\sigma},
$$

where every function is to be calculated on $\theta=\pi / 2$ and $r=R$, the radius of the geodesic circle.

The previous expression states that the nodes of nearby geodesics are separated by regular intervals of the coordinate $\phi$. If $\Omega$ is different from one, these 
nodes will travel around the geodesic circle instead of remaining at constant values of the azimuthal angle. Since it would be of interest to write down the result in a coordinate independent expression, it will be required to make use of the geodesic equations to remove the dependence on the radius of the circle, $R$, and also to cast the energy, $E$, as a function of the angular momentum of the circular orbit, $l$, so that the final result be a function of the multipole moments and $l$ only.

As it is well known, the timelike geodesic equations can be obtained from the Lagrange equations applied to a functional $L=g_{\mu \nu} \dot{x}^{\mu} \dot{x}^{\nu}$ and the constraint $L=-1$. Two of these equations, namely (34) and (35), have already been used to remove the dependence on the derivatives of the ignorable coordinates. The equation corresponding to variations of $\theta$ is automatically satisfied. We are left just with two equations, corresponding to the constraint and the variations of $r$, viz.

$$
\begin{gathered}
1=\frac{E^{2}}{f}-f \frac{(l-E A)^{2}}{r^{2}} \\
\frac{E^{2}}{f^{2}} \partial_{r} f-2 E f \frac{l-E A}{R^{2}} \partial_{r} A-f^{2} \frac{(l-E A)^{2}}{R^{4}} \partial_{r}\left(\frac{r^{2}}{f}\right)=0,
\end{gathered}
$$

where we have used $\dot{r}=0$ and $\theta=\pi / 2$.

From the last equation we get $E / l$ as a function of $R$,

$$
\begin{gathered}
\frac{E}{l}=\frac{-b \pm \sqrt{b^{2}-4 a c}}{2 a} \\
a=A^{2} f^{2} R^{-4} \partial_{r}\left(r^{2} f^{-1}\right)-f^{-2} \partial_{r} f-2 A f R^{-2} \partial_{r} A= \\
=-2 P_{0} R^{-2}+O\left(R^{-3}\right) \\
b=2 R^{-2} f \partial_{r} A-2 A f^{2} R^{-4} \partial_{r}\left(r^{2} f^{-1}\right)= \\
=-12 i P_{1} R^{-4}+O\left(R^{-5}\right) \\
c=f^{2} R^{-4} \partial_{r}\left(r^{2} f^{-1}\right)=2 R^{-3}+O\left(R^{-4}\right) .
\end{gathered}
$$

Since the energy must be positive, the solution with the minus (plus) signus in front of the square root equation corresponds to a positive (negative) $l$. On the other hand equation (73) furnishes $l$ in terms of $E / l$ which has obviously the same coefficients which were obtained for $E$ in the previous section after substitution of $E_{0}=-1 / 2$.

The frequency of the precession of the nodes can be written now independently of the choice of coordinates. As was to be expected, the first term is equal 
to one, corresponding to the frequency of the nodes when the mass distribution of the gravitational source is perfectly spherical.

The contributions to the precession of the line of nodes of a timelike geodesic which is slightly tilted with respect to a geodesic equatorial circle have been classified in the same way as it was done for the perihelion shift: A classical term plus the relativistic terms, divided according to their multipole content. In order to write down the expressions in non-geometrized units, factors including $G$ and $c$ have to be included as it was done in the previous section. The description which was made there of the necessary factors for each term in $\Delta \phi$ for the perihelion shift also applies. The information that we have of the metric allows us to calculate the coefficients up to $l^{-13}$.

There is, of course, no contribution from a pure mass monopole. Were the frequency equal to one, then the nodes would remain at constant $\phi$. Therefore, $\Delta \phi=2 \pi\left(\Omega^{-1}-1\right)$ describes the angle through which the line of nodes has precessed in one revolution of the reference circle,

$$
\begin{aligned}
\Delta \phi & =\pi\left\{\Delta_{0}+\Delta_{2}+\Delta_{4}+\Delta_{8}+\Delta_{16}+\Delta_{32}+\Delta_{2 \times 4}+\right. \\
& \left.+\Delta_{2 \times 8}+\Delta_{2 \times 16}+\Delta_{4 \times 8}\right\}
\end{aligned}
$$

For oblate objects $\left(P_{2}<0\right)$, the influence of the mass quadrupole amounts to a delay in the precession of the line of nodes with respect to the $\phi$ coordinate on the circle of reference. Therefore the line of nodes does not precess in the same direction as the perihelion. This fact should not be confused with the precession of the angular momentum vector in time, which is of course positive. The contribution from the sedecimpole term is negative for positive $P_{4}$ and the one from the sexagintaduopole is positive for positive $P_{6}$. There is also a classical coupling between $P_{4}$ and $P_{2}$. The nonlinear terms in the quadrupole moment bear a positive sign,

$$
\begin{aligned}
\Delta_{0} & =\frac{3 P_{0} P_{2}}{l^{4}}+\left(-\frac{15 P_{0}^{3} P_{4}}{2}+\frac{9 P_{0}{ }^{2} P_{2}{ }^{2}}{4}\right) l^{-8}+ \\
& +\left(\frac{105 P_{0}{ }^{5} P_{6}}{8}+\frac{45 P_{0}{ }^{4} P_{2} P_{4}}{8}+\frac{81 P_{0}{ }^{3} P_{2}{ }^{3}}{8}\right) l^{-12} .
\end{aligned}
$$

Most of the dipole-dependent terms are sensitive to the direction of motion of the test particle relative to the rotation of the source. If the angular momenta are parallel ( $l$ has the same sign as $J=-i P_{1}$ ), then the linear and cubic terms in $P_{1}$ induce an advance of the line of nodes. On the other hand the quadratic and quartic terms in the dipole moment are not sensitive to that relative sign and their influence always amounts to a delay,

$$
\Delta_{2}=-\frac{4 i P_{0} P_{1}}{l^{3}}-\frac{18 i P_{0}^{3} P_{1}}{l^{5}}+\frac{18 P_{0}{ }^{2} P_{1}{ }^{2}}{l^{6}}-\frac{243 i P_{0}^{5} P_{1}}{2 l^{7}}+\frac{4131 P_{0}^{4} P_{1}^{2}}{14 l^{8}}+
$$




$$
\begin{aligned}
& +\left(196 i P_{0}{ }^{3} P_{1}^{3}-\frac{3861 i P_{0}{ }^{7} P_{1}}{4}\right) l^{-9}+\frac{27294 P_{0}{ }^{6} P_{1}^{2}}{7} l^{-10}+ \\
& +\left(\frac{37983 i P_{0}^{5} P_{1}^{3}}{7}-\frac{268515 i P_{0}^{9} P_{1}}{32}\right) l^{-11}+ \\
& +\left(-2565 P_{0}{ }^{4} P_{1}^{4}+\frac{1060043 P_{0}^{8} P_{1}{ }^{2}}{22}\right) l^{-12}+ \\
& +\left(\frac{734200 i P_{0}{ }^{7} P_{1}^{3}}{7}-\frac{4944807 i P_{0}{ }^{11} P_{1}}{64}\right) l^{-13} .
\end{aligned}
$$

The relativistic contribution of the linear terms in the quadrupole moment $P_{2}$ is again negative for oblate sources. The quadratic terms furnish a negative contribution to the precession shift regardless of whether the source is oblate or prolate, whereas the classical quadratic correction is positive, as it was shown previously,

$$
\begin{aligned}
\Delta_{4} & =\frac{24 P_{0}{ }^{3} P_{2}}{l^{6}}+\frac{2799 P_{0}{ }^{5} P_{2}}{14 l^{8}}+\left(-24 P_{0}{ }^{4} P_{2}{ }^{2}+\frac{12396 P_{0}{ }^{7} P_{2}}{7}\right) l^{-10}+ \\
& +\left(-\frac{327223 P_{0}{ }^{6} P_{2}{ }^{2}}{308}+\frac{361993 P_{0}{ }^{9} P_{2}}{22}\right) l^{-12}
\end{aligned}
$$

As it happened with the perihelion precession, the rotational octupole term has the opposite sign to the one of the linear dipole term and of course it is dependent on the direction in which the probe orbits,

$$
\Delta_{8}=\frac{12 i P_{0}^{3} P_{3}}{l^{7}}+\frac{156 i P_{0}{ }^{5} P_{3}}{l^{9}}+\frac{3387 i P_{0}{ }^{7} P_{3}}{2 l^{11}}+\frac{17707 i P_{0}{ }^{9} P_{3}}{l^{13}} .
$$

The relativistic sedecimpole term bears the same sign as its classical counterpart, although it is much smaller in magnitude. There is no relativistic term in $P_{6}$, just the classical term which has already been described,

$$
\Delta_{16}=-\frac{120 P_{0}^{5} P_{4}}{l^{10}}-\frac{2905 P_{0}^{7} P_{4}}{2 l^{12}},
$$

and again we have another permutation of sign; the term in $P_{5}$ bears the same sign as the linear dipole term,

$$
\Delta_{32}=-\frac{45 i P_{0}{ }^{5} P_{5}}{2 l^{11}}-\frac{1905 i P_{0}{ }^{7} P_{5}}{4 l^{13}} .
$$

Now we discuss the coupling terms between multipole moments other than the mass. The bilinear coupling between the dipole and the quadrupole moment is positive for oblate gravitational sources which rotate in the same direction 
as the test particle. The quadratic term in $P_{1}$ is negative for oblate objects no matter in which direction they rotate. The quadratic term in $P_{2}$ is positive when the angular momenta $J$ and $l$ are parallel,

$$
\begin{aligned}
\Delta_{2 \times 4} & =\frac{18 i P_{0}{ }^{2} P_{1} P_{2}}{l^{7}}+\frac{441 i P_{0}{ }^{4} P_{1} P_{2}}{l^{9}}-\frac{273 P_{0}{ }^{3} P_{1}{ }^{2} P_{2}}{l^{10}}+ \\
& +\left(-\frac{81 i P_{0}{ }^{3} P_{1} P_{2}{ }^{2}}{2}+\frac{198369 i P_{0}{ }^{6} P_{1} P_{2}}{28}\right) l^{-11}-\frac{3007003 P_{0}{ }^{5} P_{1}{ }^{2} P_{2}}{308 l^{12}}+ \\
& +\left(-\frac{10899 i P_{0}{ }^{5} P_{1} P_{2}{ }^{2}}{4}-4482 i P_{0}{ }^{4} P_{1}{ }^{3} P_{2}+\frac{5509583 i P_{0}{ }^{8} P_{1} P_{2}}{56}\right) l^{-13}(86)
\end{aligned}
$$

The rotational bilinear coupling between the dipole and the octupole moment is independent of the direction of rotation and it is positive when $J=-i P_{1}$ and $J_{3}=-i P_{3}$ have the same sign. There is however a higher coupling which is quadratic in the dipole moment and contributes in the same way as $\Delta_{8}$ does,

$$
\Delta_{2 \times 8}=-\frac{186 P_{0}{ }^{4} P_{1} P_{3}}{l^{10}}-\frac{100201 P_{0}{ }^{6} P_{1} P_{3}}{22 l^{12}}-\frac{3168 i P_{0}{ }^{5} P_{1}{ }^{2} P_{3}}{l^{13}} .
$$

The last terms to be considered up to this order are the couplings between $P_{1}$ and $P_{4}$ and between $P_{2}$ and $P_{3}$, which are both sensitive to the relative directions of rotation of the source and the probe particle,

$$
\begin{aligned}
\Delta_{2 \times 16} & =-\frac{255 i P_{0}{ }^{4} P_{1} P_{4}}{2 l^{11}}-\frac{14193 i P_{0}{ }^{6} P_{1} P_{4}}{4 l^{13}} \\
\Delta_{4 \times 8} & =-\frac{21 i P_{0}{ }^{4} P_{2} P_{3}}{l^{11}}-\frac{1971 i P_{0}{ }^{6} P_{2} P_{3}}{2 l^{13}} .
\end{aligned}
$$

\section{Conclusions}

In this paper we have displayed the approximate general asymptotically flat stationary axisymmetric metric for the vacuum spacetime surrounding a compact source possessing a symmetry plane orthogonal to the symmetry axis. The calculations have been carried out for the Ernst potential up to the term in $r^{-7}$ in the pseudospherical radial coordinate. This has been useful to calculate relativistic corrections to the classical orbits around a compact mass distribution. In particular the perihelion precession on the symmetry plane and the precession of the nodes of a slightly tilted circular orbit have been calculated.

Concerning the perihelion shift, it has been shown that the contributions of each gravitational and rotational multipole moment follow a curious pattern of alternation of signs: The term in the mass monopole is always positive, whereas the linear quadrupole term is negative for positive $P_{2}$ (the quadratic term in 
$P_{2}$ is always positive) and the sedecimpole term is again positive for positive $P_{4}$. The sexagintaquattuorpole $P_{6}$ is outside the limits of our perturbative expansion. If we think for instance of an oblate source which is very close to a classical axisymmetric homogeneous ellipsoid, then we would also have alternating signs in the gravitational multipole expansion $\left(P_{2 n}=3 P_{0}\left(c^{2}-\right.\right.$ $\left.\left.a^{2}\right)^{n} /[(2 n+1)(2 n+3)]\right), c$ and $a$ being the lengths of the ellipsoid's semiaxis parallel and orthogonal to the symmetry axis, respectively) and every term would have a positive contribution. The linear and cubic rotational dipole term is always positive for counterrotating configurations of the source and the probe (the quadratic contribution is always positive regardless of the relative rotation) whereas the octupole term is positive if $J_{3}=-i P_{3}$ and $l$ bear the same sign. The trigintaduopole term has the same sign as the dipole term. It should be pointed out that the energy dependent terms are not strong enough to affect these signs. The coupling between different multipoles preserves the sign of the product of the corresponding linear terms, that is, if there is a $P_{n}-P_{m}$ coupling, then its sign is obtained from the product of the -1 factors multiplying the corresponding linear terms in $P_{n}$ and $P_{m}$. No coupling between mass multipole moments of order higher than the monopole appears to this order except for self-couplings. The sign independent terms, such as the mass monopole term and the quadratic dipole and quadratic quadrupole terms, always give rise to a perihelion advance.

The influence of the different multipole moments on the precession of the line of nodes of a particle departing by a small amount from the equatorial plane is somewhat different from the behaviour which has been shown for the perihelion precession: The alternating pattern of the signs of the linear terms in the multipole moments other than the mass is preserved both for gravitational and rotational moments independently up to the considered perturbation order: If the $P_{2 n}$ were all positive, then the contribution of each $P_{2 n}$ would be opposite to the one of $P_{2 n+2}$ and a similar reasoning is valid for the rotational terms. However the relativistic coupling terms bear the opposite sign to the one which would be expected from the product of the -1 factors before the corresponding linear terms: For instance, if the $P_{1}$ and $P_{2}$ linear terms are positive then the corresponding bilinear coupling is negative. On the other hand, this rule is not valid for the Newtonian self-couplings. Another difference arises from the fact that the gravitational sexagintaduopole moment $P_{6}$ does influence the precession of the line of nodes in this order of perturbation, whereas it does not affect the perihelion precession. Also, there were no couplings between the gravitational moments (self-couplings and mass-couplings excluded) in the perihelion precession, but there is one (the classical $P_{2}-P_{4}$-coupling) in the node precession. It is curious that, while the Newtonian sign-independent terms are always positive, the relativistic ones (the quadratic dipole and the quadratic quadrupole self-couplings) are negative.

Of course most of these corrections are meaningless for astronomical purposes in our solar system, but are very likely to be relevant for highly relativistic astrophysical objects, suchs as pulsars and blackholes, where other post-Newtonian effects [5] have been shown to be present. 


\section{A Schwarschild spacetime}

In this appendix we shall study the range of applicability of the perturbation expansion for bounded orbits in Schwarschild's spacetime. For this metric the geodesic equation (38) can be solved exactly in terms of elliptic functions. Instead of writing it as a differential equation for $u$ as a function of $\phi$, we are writing it as a differential equation for $\phi$. In this section $u$ is no longer the inverse of the pseudospherical radius but of the usual Boyer-Lindquist radius,

$$
\phi_{u}=\left(2 P_{0} u^{3}-u^{2}+\frac{2 P_{0}}{l^{2}} u+\frac{E^{2}-1}{l^{2}}\right)^{-1 / 2}=g(u)^{-1 / 2} .
$$

We are considering that $E<1$ and therefore $g(u)$ has at least one zero. For bounded motion we need three zeros so that the orbit ranges between the apsidal points. If we call these zeros $a \geq b \geq c \geq 0$, then equation (90) can be integrated 11] in terms of the elliptic integral of the first kind, $F(\gamma, q)$, in the region $b \geq u>c$,

$$
\begin{gathered}
\left(\phi-\phi_{0}\right) \sqrt{\frac{P_{0}(a-c)}{2}}=F(\gamma, q)=\int_{0}^{\gamma} d \alpha\left(1-q^{2} \sin ^{2} \alpha\right)^{-1 / 2} \\
\gamma=\arcsin \sqrt{\frac{u-c}{b-c}} \quad q=\sqrt{\frac{b-c}{a-c}} .
\end{gathered}
$$

An expression for $u$ in terms of $\phi$ is easily obtained taking into account that the elliptic sine is the sine of $F(\gamma, q)$,

$$
u=c+(b-c) \operatorname{sn}^{2}\left\{\sqrt{\frac{P_{0}(a-c)}{2}}\left(\phi-\phi_{0}\right)\right\} .
$$

Since the real period of the elliptic sine is $4 K(q)=4 F(\pi / 2, q)$, then our $u$ function is $2 K(q)$-periodic. Therefore the exact perihelion precession of the orbit of a test particle around a spherical non-rotating compact object will be,

$$
\Delta \phi=\frac{2 \sqrt{2} K(q)}{\sqrt{P_{0}(a-c)}}-2 \pi,
$$

the perturbation expansion of which in $\epsilon$ coincides with the one of $\Delta_{1}$ in equation (59), as it was to be expected,

$$
K(q)=\frac{\pi}{2}\left\{1+\sum_{n=1}^{\infty}\left(\frac{(2 n-1) ! !}{2^{n} n !}\right)^{2} q^{2 n}\right\} .
$$

The limits of the range of applicability of the previous expansion are $q=1$ $(a=b)$ and $q=0(b=c)$. For both values, there are two zeros of $g(u)$ which coalesce and the bounded motion is no longer stable. Therefore we are led to study the range of parameters for which $g(u)$ has double roots. This happens when $u$ takes either of the values $u_{ \pm}$which are solutions of $g^{\prime}(u)=0$, 


$$
u_{ \pm}=\frac{1 \pm \sqrt{1-12 \epsilon^{2}}}{6 P_{0}}
$$

and therefore the allowed region is the one enclosed between the curves $g\left(u_{ \pm}\right)=$ 0 in the $E^{2}-q^{2}$ parameter plane. This yields critical values for the energy per unit of mass, $E_{c}^{2}=8 / 9$, and the perturbation parameter, $\epsilon_{c}^{2}=1 / 12$. The perturbative approach is no longer valid beyond this point in the parameter plane since there are no stable bounded orbits. It is remarkable that for $\epsilon^{2}>$ $1 / 16$ there is not only a lower limit for the energy of the bounded orbit but also an upper limit.

\section{B Classical precession of the line of nodes}

In this appendix we shall briefly derive the classical expression for the precession of the line of nodes.

The Lagrangian for the motion of a particle in a gravitational field is,

$$
\begin{gathered}
L=\frac{1}{2} \dot{r}^{2}+\frac{1}{2} r^{2} \dot{\theta}^{2}+\frac{1}{2} r^{2} \sin ^{2} \theta \dot{\phi}^{2}-V(r, \theta) \\
V(r, \theta)=-\sum_{n=0}^{\infty} \frac{P_{n} p_{n}(\cos \theta)}{r^{n+1}} .
\end{gathered}
$$

The equations of motion and conserved quantities which can be obtained from this Lagrangian are:

$$
\begin{gathered}
E=\frac{1}{2} \dot{r}^{2}+\frac{1}{2} r^{2} \dot{\theta}^{2}+\frac{1}{2} r^{2} \sin ^{2} \theta \dot{\phi}^{2}+V(r, \theta) \\
l=r^{2} \sin ^{2} \theta \dot{\phi} \\
\ddot{r}=r \dot{\theta}^{2}+r \sin ^{2} \theta \dot{\phi}^{2}-\partial_{r} V(r, \theta) \\
r^{2} \ddot{\theta}+2 r \dot{r} \dot{\theta}=r^{2} \sin \theta \cos \theta \dot{\phi}^{2}-\partial_{\theta} V(r, \theta) .
\end{gathered}
$$

Truncating the Legendre expansion at the sexagintaquattuorpole multipole moment, $P_{6}$, we get the following expressions for the energy per unit of mass, $E$, and the radius, $R$, of a circular orbit on the plane $\theta=\pi / 2$ in the far field region,

$$
\begin{array}{r}
E=-\frac{P_{0}^{2}}{2 l^{2}}+\frac{P_{0}^{3} P_{2}}{2 l^{6}}-\frac{3 P_{0}^{5} P_{4}+9 P_{0}^{4} P_{2}^{2}}{8 l^{10}}+O\left(l^{-14}\right) \\
R^{-1}=\frac{P_{0}}{l^{2}}-\frac{3 P_{0}^{2} P_{2}}{2 l^{6}}+\frac{15 P_{0}^{4} P_{4}+36 P_{0}^{3} P_{2}^{2}}{8 l^{10}}+O\left(l^{-14}\right) .
\end{array}
$$


In order to obtain the oscillations about the equatorial plane of a slightly tilted bounded trajectory with respect to the circular orbit, we introduce a small variation in the equation (102). The result will be divided by $\dot{\phi}^{2}$ to yield the evolution of $\delta \theta$ as a function of $\phi$,

$$
(\delta \theta)_{\phi \phi}=-\Omega^{2} \delta \theta \quad \Omega=\sqrt{1+\frac{R^{2}}{l^{2}} V_{\theta \theta}(R, \pi / 2)} .
$$

From this expression we get the frequency of the oscillations, $\Omega$, which can be written in terms of $l$ and the multipole moments by inserting equation (104) into it,

$$
\begin{aligned}
\Delta \phi & =2 \pi\left(\frac{1}{\Omega}-1\right)=\frac{3 \pi P_{0} P_{2}}{l^{4}}+\frac{\pi\left(9 P_{0}^{2} P_{2}^{2}-30 P_{0}^{3} P_{4}\right)}{4 l^{8}}+ \\
& +\frac{\pi\left(105 P_{0}^{5} P_{6}+45 P_{0}^{4} P_{2} P_{4}+81 P_{0}^{3} P_{2}^{3}\right)}{8 l^{12}}+O\left(l^{-16}\right)
\end{aligned}
$$

which obviously coincides with the term $\Delta_{0}$ which was calculated using the full relativistic theory in section IV

\section{Acknowledgements}

The present work has been supported by Dirección General de Enseñanza Superior Project PB98-0772. L.F.J. wishes to thank F.J. Chinea, L.M. GonzálezRomero, F. Navarro-Lérida and M.J. Pareja for valuable discussions. L.F.J. wishes to thank the Department of Mathematical Sciences of the Loughborough University of Technology for their hospitality.

\section{References}

[1] Solutions of Einstein's equations: Techniques and Results (eds.: C. Hoenselaers and W. Dietz, Springer Verlag), Berlin-New York (1984)

[2] R. Geroch, J. Math. Phys. 112580 (1970)

[3] R. O. Hansen, J. Math. Phys. 1546 (1974)

[4] G. Fodor, C. Hoenselaers, Z. Perjés, J. Math. Phys. 302252 (1989)

[5] T. D'amour, G. Schäfer, Nuovo Cimento B 101127 (1988)

[6] C. Hoenselaers, Prog. Theor. Phys. 56324 (1977)

[7] H. Quevedo, Ph. D. Thesis Universität zu Köln (1987)

[8] F. J. Ernst, Phys.Rev. 1671175 (1968)

[9] H. Goldstein, Classical Mechanics, Addison-Wesley (1980) 
[10] V. Perlick, Class. Quantum Grav. 91009 (1992)

[11] I. S. Gradsteyn, I. M. Ryzhik, Table of integrals, series and products, Academic Press, New York (1965) 